\title{
Extrapulmonary Tuberculosis Presenting as a Suspected Case of Metastatic Breast Cancer
}

\author{
Sonia Almeida, Marta Valentim, Catarina Neto, Marta Cerol, Marina Boticário, Maria Ines Santos, Ana Gameiro \\ Hospital Distrital De Santarem E.P.E., Santarém, Portugal
}

\section{Doi: 10.12890/2019_001062 - European Journal of Case Reports in Internal Medicine - @ EFIM 2019}

Received: 29/01/2019

Accepted: $13 / 02 / 2019$

Published: 22/03/2019

\begin{abstract}
How to cite this article: Almeida S, Valentim M, Neto C, Cerol M, Boticário M, Santos MI, Gameiro A. Extrapulmonary tubercolosis presenting as a suspected case of metastasic breast cancer. EJCRIM 2019;6: doi:10.12890/2019_001062.
\end{abstract}

Conflicts of Interests: The Authors declare that there are no competing interests.

This article is licensed under a Commons Attribution Non-Commercial 4.0 License

\section{ABSTRACT}

Extrapulmonary tuberculosis (TB) represents a diagnostic challenge. Bone TB is an uncommon and important presentation of extrapulmonary TB, which can lead to bone destruction, deformity and even paraplegia. Breast TB is rare and often confused with neoplasia, since the clinical and imaging presentations are not specific. Any of these extrapulmonary TB presentations, in the absence of cultural isolation of mycobacteria, oblige the exclusion of other diseases (secondary or infectious diseases). The authors report a case of multifocal extrapulmonary TB, as an example of the problem with the differential diagnosis of the disease.

\section{LEARNING POINTS}

- Extrapulmonary TB remains a diagnostic challenge.

- Consider breast TB as a differential diagnosis of mammary nodules.

- A high level of suspicion is necessary to make an early diagnosis and start appropriate treatment before irreversible damage takes place.

\section{KEYWORDS}

Infectious diseases, tuberculosis, extrapulmonary tuberculosis

\section{CASE DESCRIPTON}

A 72-year-old woman presenting with fatigue, low back pain, fever, night sweats and weight loss for the last 2 years was admitted to the emergency department. She was febrile, pale and presented with a mammary nodule in the inferior-external quadrant of the left breast with redness, oedema and retraction of the suprajacent cutaneous tissue.

Laboratory findings showed a normocytic and normochromic anaemia (haemoglobin $10.4 \mathrm{~g} / \mathrm{dL}$ ), C-reactive protein levels of $11.5 \mathrm{mg} / \mathrm{dL}$ and an erythrocyte sedimentation rate of $70 \mathrm{~mm} / \mathrm{h}$.

Mammography revealed increased echogenicity of the mammary tissue, translated in ultrasound as an area of structural heterogeneity with interstitial oedema, without nodular formations (Fig. 1).

The patient underwent a full body CT scan and a nodular lesion in the left breast could be seen (Fig. 2). A CT scan of the lumbar spine was also performed (Fig. 3) presenting extensive lytic lesions in the central and posterior region of the L1 body, conditioning deformation of the dural sac and extensive erosions of the L2 vertebral platform, associated with fracture and sagging of the vertebral platform with slight retreat of the posterior wall. These findings were suggestive of metastatic lesions, but it was not possible to exclude spondylodiscitis. Bone scintigraphy revealed no other significant alterations. Blood cultures, viral serologies, tumour markers and adenosine deaminase were all within the reference values. 
Fine needle aspiration biopsy (FNA) of the breast lesion and paravertebral mass biopsy evidenced granulomatous lesions with central necrosis, and therefore, the diagnosis of tuberculosis (TB) was the most probable. Cultural examinations and Acid-Fast Bacilli (AFB) testing in the biopsy specimens and in blood cultures did not identify the presence of mycobacteria.

Despite the absence of mycobacteria, the diagnosis of extrapulmonary TB (mammary and lumbar vertebrae) was presumed, with the patient started on antimycobacterial therapy (rifampicin, isoniazid, pyrazinamide and ethambutol) with progressive improvement of pain, resolution of functional disability, imaging improvement of bone lesions (Fig. 4) and complete resolution of mastitis.

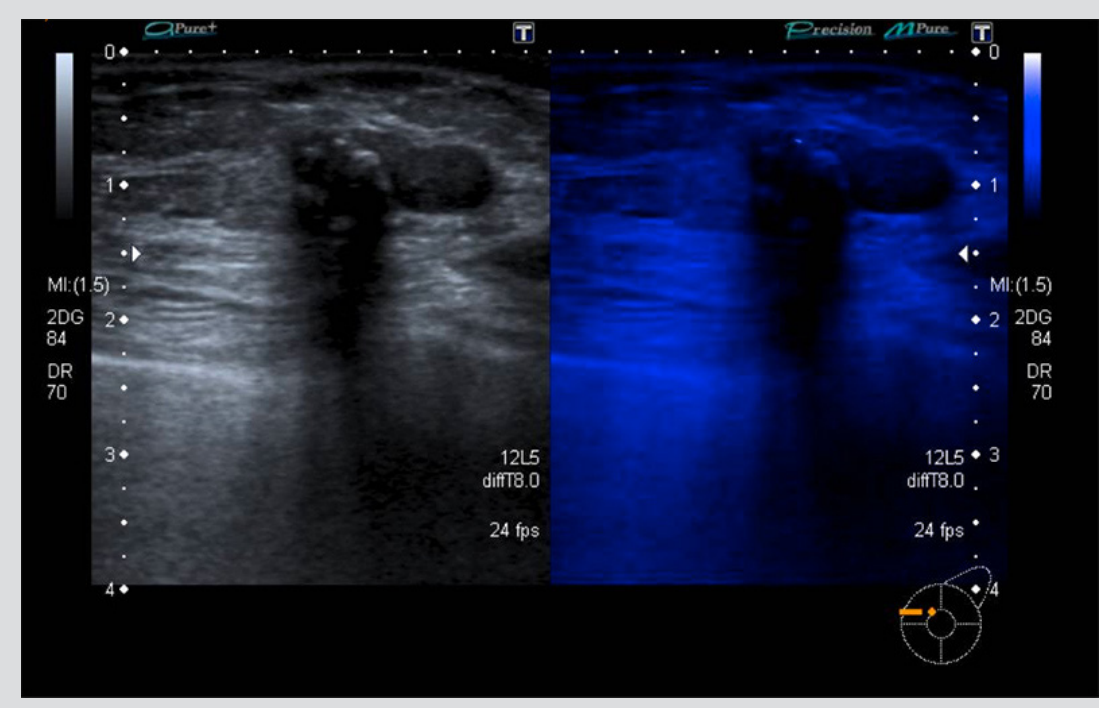

Figure 1. Breast ultrasound: area of structural heterogeneity in the left breast, thickening of the skin and swelling in the lower quadrants with interstitial oedema, without nodular formations.

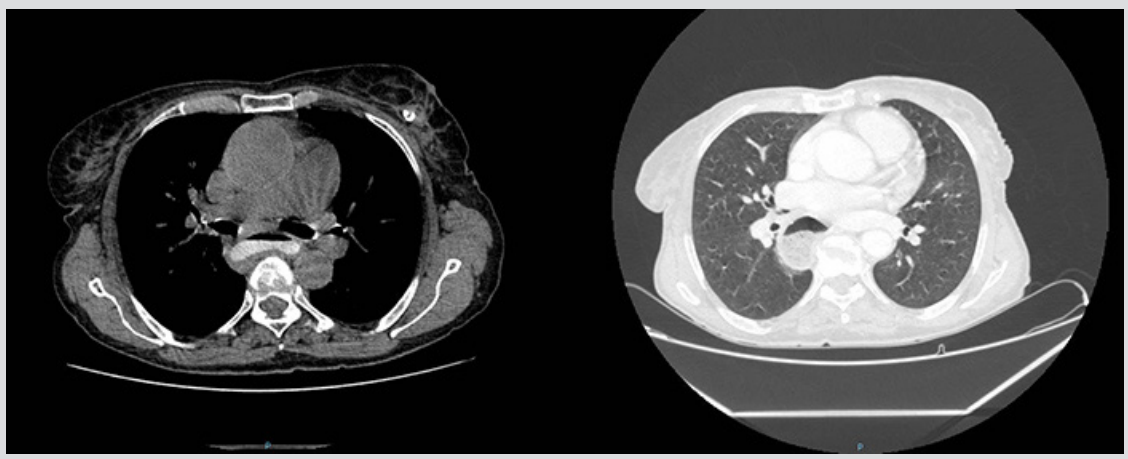

Figure 2. Chest CT scan: nodular lesion in the left breast.
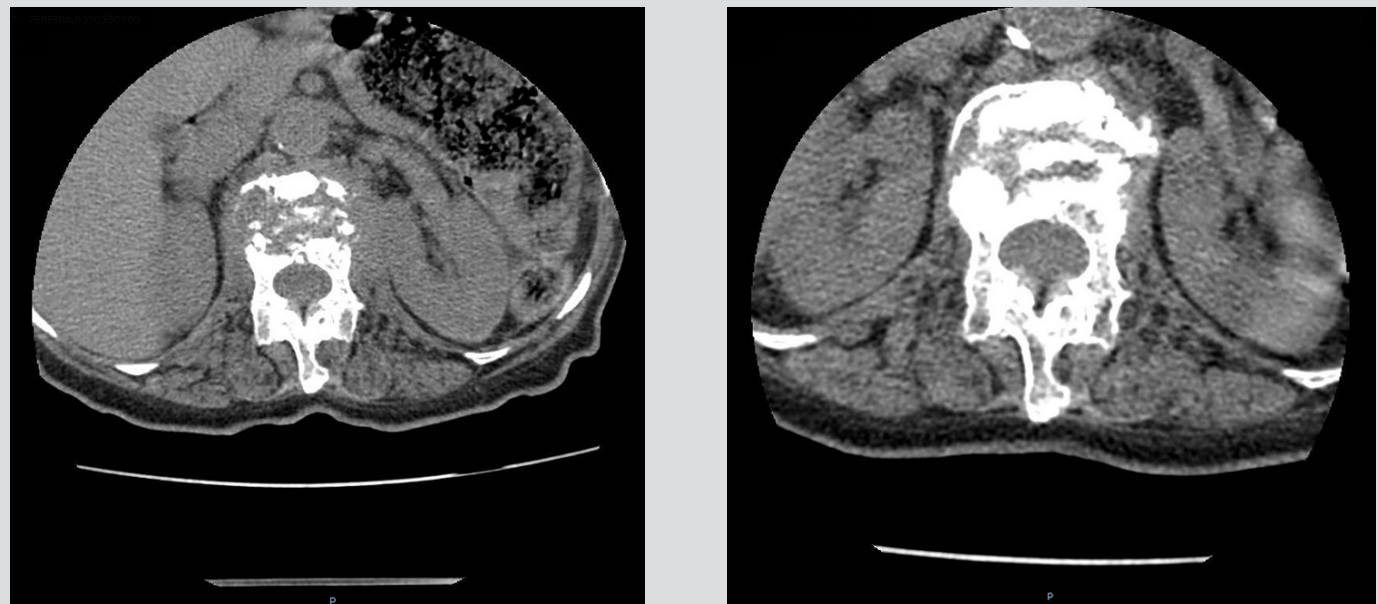

Figure 3. Lumbar spine CT scan: extensive lytic lesions in the central and posterior region of the L1 body, conditioning deformation of the dural sac.

Figure 4. Lumbar CT scan after antimycobacterial therapy: improvement of bone lesions. 


\section{DISCUSSION}

TB remains a major public health problem worldwide. According to the Global Tuberculosis Report 2018, it affected 10 million people in 2017 and is responsible for 1.2-1.4 million deaths globally in HIV-negative patients ${ }^{[1]}$. It is caused by Mycobacterium tuberculosis and most often affects the lungs. About $23 \%$ of the world's population (1.7 billion people) has latent tuberculosis, with a risk of reactivation of $5-15 \%{ }^{[1]}$. In the literature, bone TB represents $10-20 \%$ of cases of extrapulmonary TB and 1-2\% of all cases of TB ${ }^{[2,3]}$. The spine is involved in up to $50 \%$ of cases of bone TB and the dorso-lumbar region of the spine is the most frequently affected place ${ }^{[4]}$, as in the case described here. It usually results from the haematogenous spread of mycobacteria in the dense vasculature of the bone of the vertebral bodies. The presentation is usually slowly progressive and nonspecific, which may delay the diagnosis. Imaging studies are essential to determine the degree of spinal involvement ${ }^{[5-7]}$. Early diagnosis of bone disease and appropriate treatment are essential to minimise the risk of deformity and improve prognosis ${ }^{[8]}$.

TB of the breast was first described in $1829^{[9]}$, and it remains a very rare presentation of extrapulmonary TB, with an incidence of $0.06-1 \%$ of cases in developed countries and up to $4.5 \%$ of cases in endemic countries ${ }^{[9,10]}$. It is a rare entity due to the high resistance offered by breast tissue to the survival and multiplication of TB bacilli ${ }^{[11}$. The most common form of clinical presentation is a single and unilateral solid lesion, with poorly defined and hard-consistency borders, centrally located or in the supero-external quadrants of the breast ${ }^{[10,12]}$, which may hinder the distinction between this entity and breast carcinoma.

Radiological examinations can also mimic neoplastic disease, since there are no specific mammographic signs of breast TB[11,12]. Mammography may show irregular heterogeneous opacities, poorly delimited and sometimes with the presence of calcifications, suggesting a malignant aetiology, whereas on ultrasound it often appears as a hypoechogenic and heterogeneous image with minimal posterior reinforcement ${ }^{[13]}$. The diagnosis is ideally performed by isolating the mycobacteria in the mammary tissue, either by culture or by ZiehlNeelsen staining. However, since the disease is typically paucibacillary, the mycobacteria are isolated in only about $25 \%$ of cases ${ }^{[14]}$. FNA biopsy is an important diagnostic tool, since more than $70 \%$ of the cases can be diagnosed by evidence of typical necrotising granulomatous lesions ${ }^{[15,16]}$, as happened in the case described above.

TB is a treatable and curable disease, following a schedule of antimycobacterial therapy with at least 4 drugs for at least 6 months, even in cases of extrapulmonary $\mathrm{TB}^{[1,17,18]}$. For breast TB surgery is also a valid treatment (abscesses refractory to antimycobacterial therapy).

Extrapulmonary TB remains a diagnostic challenge in the current population. The authors described a case that could lead to the suspicion of neoplastic disease with breast involvement and bone metastasis, stressing the importance of the diagnostic process and the accomplishment of appropriate examinations, as the prompt onset of antimycobacterial therapy is essential.

\section{REFERENCES}

1. World Health Organization. Global tuberculosis report 2018. Geneva: World Health Organization; 2018.

2. Davidson PT, Horowitz I. Skeletal tuberculosis. A review with patient presentations and discussion. Am J Med 1970;48:77-84

3. Chauhan A, Gupta BB. Spinal tuberculosis. Indian Acad Clin Med 2007;8:110-114.

4. Leonard MK, Blumberg HM. Musculoskeletal tuberculosis. Microbiol Spectr 2017;5:371-392

5. Gautam MP, Karki P, Rijal S, Singh R. Pott's spine and paraplegia. JNMA J Nepal Med Assoc 2005;44:106-115.

6. Nussbaum ES, Rockswold GL, Bergman TA, Erickson DL, Seljeskog EL. Spinal tuberculosis: A diagnostic and management challenge. J Neurosurg 1995;83:243-247.

7. Ansari S, Amanullah MF, Ahmad K, Rauniyar RK. Pott's spine: Diagnostic imaging modalities and technology advancements. N Am J Med Sci 2013;5:404-411.

8. Tuli SM. Historical aspects of Pott's disease (spinal tuberculosis) management. Eur Spine J 2013;22:S529-S538.

9. Cooper A. Illustration of the diseases of the breast: Part I. Longmans, Orme, London: Brown \& Green; 1829, p. 73.

10. Tewari M, Shukla HS. Breast tuberculosis: diagnosis, clinical features, and management. Indian J Med Res 2005;122:103-110.

11. Shinde SR, Chandawarkar RY, Deshmukh SP. Tuberculosis of the breast masquerading as carcinoma: A study of 100 patients. World J Surg 1995;19:379-381.

12. McKeown KC, Wilkinson KW. Tuberculosis of the breast. Br J Surg 1952;39:420-429.

13. Khanna R, Prasanna GV, Gupta P, Kumar M, Khanna S, Khanna AK. Mammary tuberculosis: report on 52 cases. Postgrad Med J 2002;78:422-424.

14. Banerjee A, Green B, Burke M. Tuberculous and granulomatous mastitis. Practitioner 1989;233:754-756.

15. Bisht SP, Gupta RJ, Khare P, Kishore B. Fine needle aspiration cytology in the diagnosis of inflammatory lesions of the breast with emphasis on tubercular mastitis. J Cytol 2007;24:155-156.

16. Kakkar S, Kapila K, Singh MK, Verma K. Tuberculosis of the breast. A cytomorphologic study. Acta Cytol 2000;44:292-296.

17. Ad Hoc Committee of the Scientific Assembly on Microbiology, Tuberculosis, and Pulmonary Infections. Tuberculosis commentary - treatment of tuberculosis and tuberculosis infection in adults and children. Clin Infect Dis 1995;21:9-27.

18. Sia IG, Wieland ML. Current concepts in the management of tuberculosis. Mayo Clin Proc 2011;86:348-361. 